\title{
Etude des bactériophages provenant des sérums de fromagerie
}

\author{
par \\ Bernadette BOISSONNET, J. Y. BOZIO, Marie-Claude LECLERC \\ et J.P. LARPENT*
}

L'insensibilité de certaines souches de streptocoques lactiques mésophiles peut s'expliquer par diverses hypothèses [7] : les phages susceptibles d'attaquer les souches ne sont pas encore en notre possession ; les phages peuvent ne pas se fixer sur les bactéries hôtes ce qui nécessiterait de déterminer des groupes d'adsorption ; les souches difficilement typables peuvent être lysogènes ou immunes vis-à-vis des phages testés ; enfin, les streptocoques difficilement typables pourraient posséder des systèmes de restriction-modification très forts qui éliminent toute possibilité de replication des virus.

C'est à ces hypothèses que nous nous sommes efforcés de répondre. Les travaux de Boissonnet [1] ont montré qu'effectivement il existait des souches de streptocoques résistantes $(10$ p. 100) à tous les phages en notre possession. Nous nous intéresserons donc aux trois hypothèses restantes : adsorption, lysogénie, restriction-modification.

\section{MATERIEL ET METHODES}

Nous avons utilisé 38 souches de S. cremoris, 39 cultures de $S$. lactis et 17 souches de $S$. diacetylactis, 86 bactériophages de provenances diverses (collections ou extraits de sérums de fromagerie) ont servi à nos tests. Les expériences ont été réalisées en milieu Mac Kay [6].

\section{RESULTATS}

1. Les résultats obtenus avec tous les groupes de phages définis par test de lysotypie sur nos souches montrent que l'adsorption est identique, que la souche soit résistante ou qu'elle soit sensible. Même avec une adsorption de 99 p. 100, certaines souches restent

* Laboratoire de Microbiologie, 4, rue Ledru - 63000 Clermont-Ferrand. 


\section{TABLEAU 1}

Pourcentage d'adsorption après un contact de $15 \mathrm{~min}$ des groupes de phages avec diverses bactéries

\begin{tabular}{c|c|c}
\hline Test de lysotypie & Bactéries & \% d'adsorption \\
\cline { 2 - 2 } Phage 1+ & Streptococcus cremoris & 66 \\
& & 76 \\
Phage 2- & Streptococcus cremoris & 60 \\
& & 94 \\
Phage 1 & Streptococcus diacetylactis & 76 \\
Phage 2- & & 90 \\
Phage 1 & Streptococcus diacetylactis & 56 \\
& Streptococcus lactis & 80 \\
Phage 2- & & 98 \\
& Streptococcus lactis & 90 \\
& & 99 \\
& & 99 \\
\end{tabular}

résistantes (tab. 1). Selon les conditions du milieu, tous les streptocoques lactiques que nous avons étudiés sont capables d'adsorber les phages. Pour toutes les souches testées, positives ou négatives en lysotypie, les pourcentages d'adsorption sont sensiblement comparables. La résistance ou la sensibilité des souches de streptocoques lactiques n'est donc pas due à l'adsorption plus ou moins forte des phages sur les bactéries.

2. De nombreux auteurs ont démontré que certaines bactéries lactiques lysogènes peuvent devenir immunes $[3,5]$. La mitomycine à la concentration de $0,3 \mu \mathrm{g} / \mathrm{ml}$ est un agent puissant de l'induction de la virulence.

La révélation des particules virales se fait avec deux souches indicatrices mises en évidence par Bozio [2] et Leclerc [4]. Sur quatorze cultures de streptocoques lactiques testées, neuf se sont révélées lysogènes et les courbes d'induction en présence de mitomycine illustrent la lyse des cultures bactériennes (fig. 1, 2, 3). $S$. cremoris, $S$. diacetylactis, $S$. lactis possèdent des souches lysogènes. 


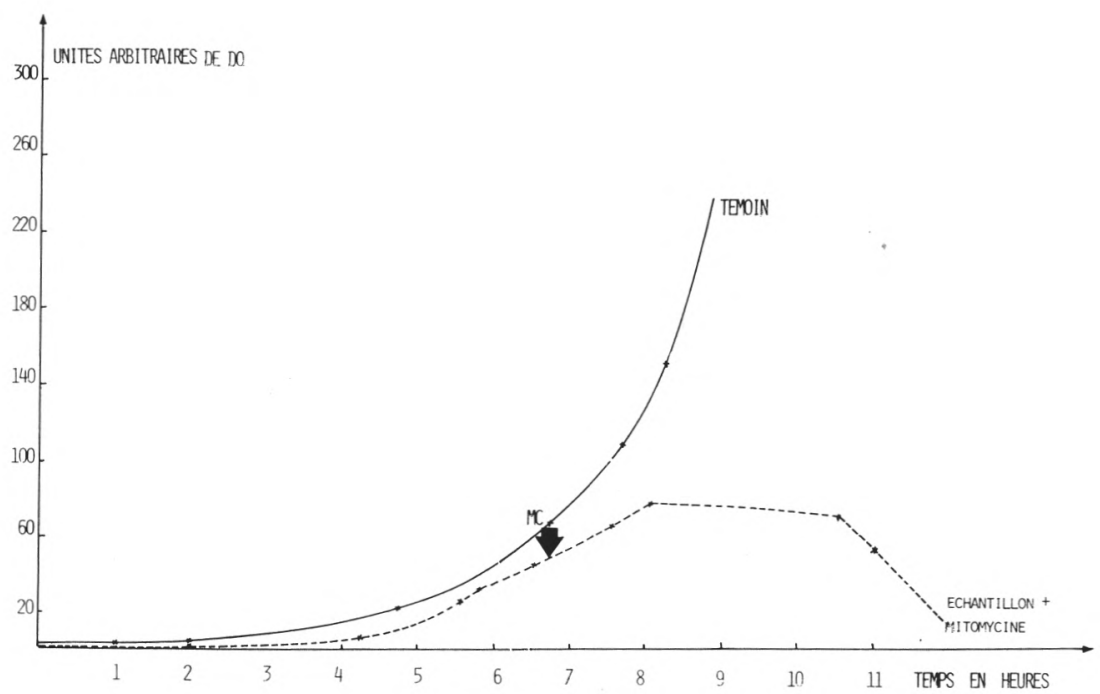

fig. 1

Courbe de croissance de Streptococcus cremoris sans addition et après addition de mitomycine.

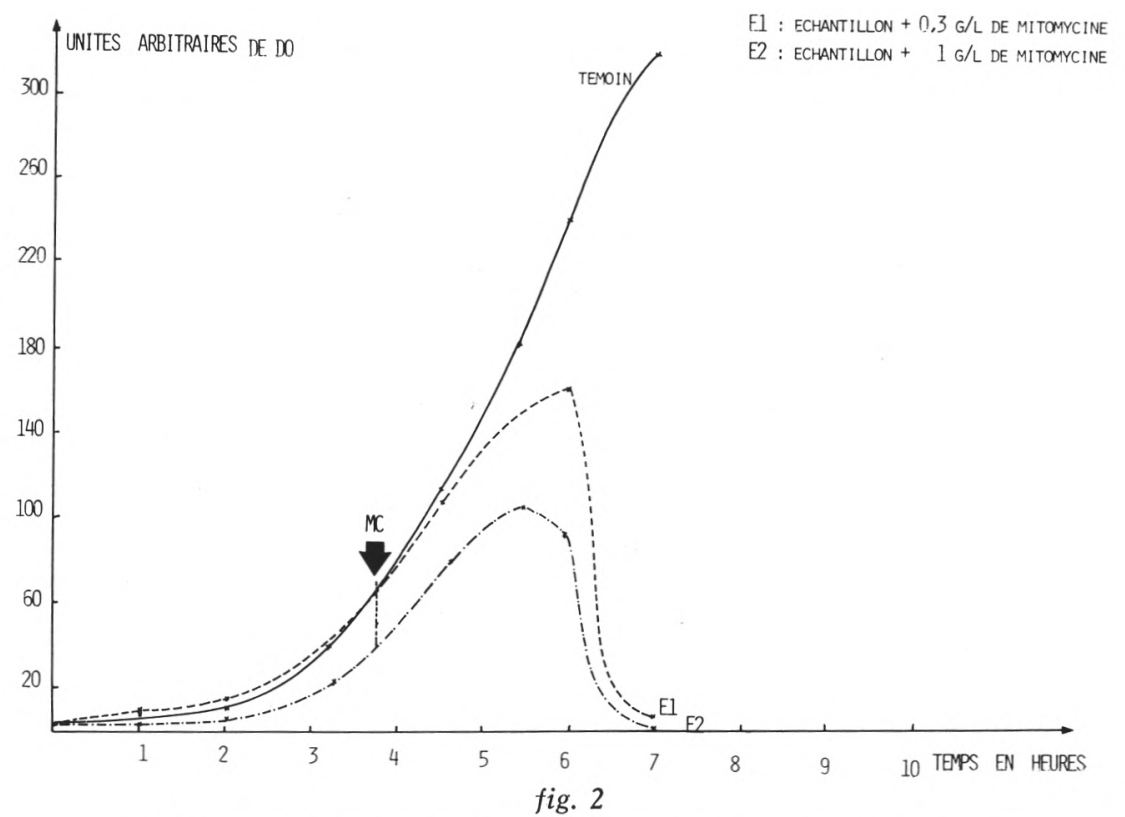

Courbe de croissance de Streptococcus diacetylactis sans addition et après addition de mitomycine. 


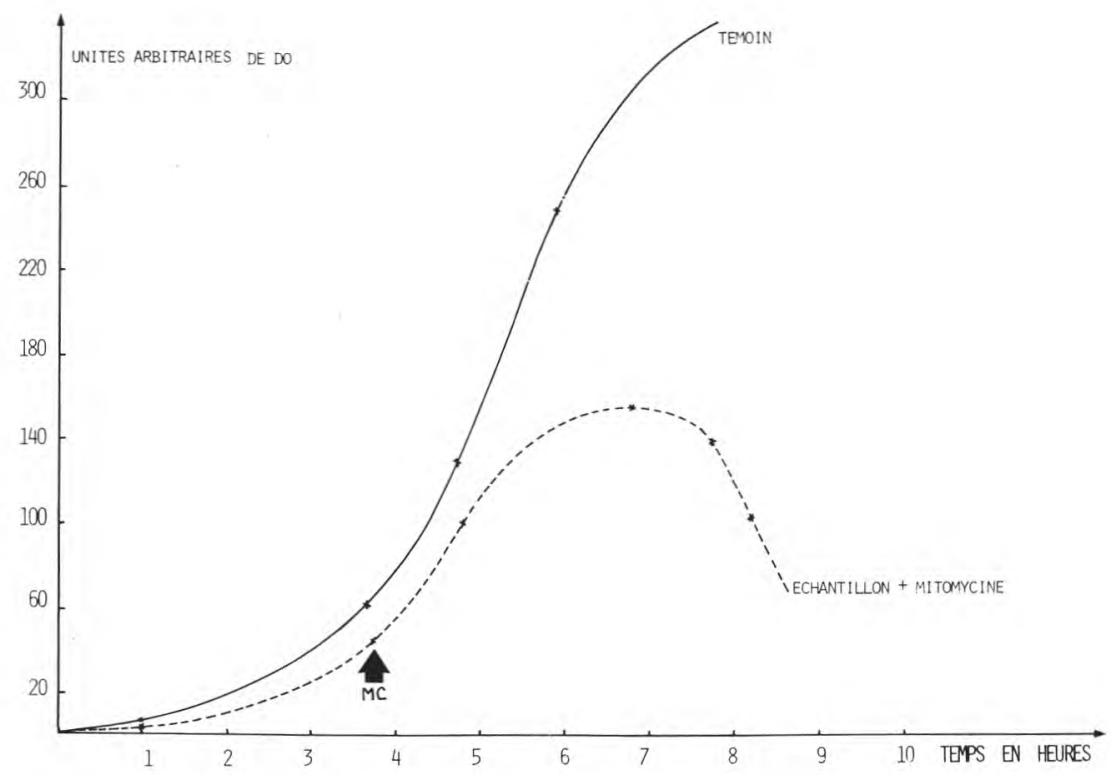

fig. 3

Courbe de croissance de Streptococcus lactis sans addition et après addition de mitomycine.

La résistance de deux souches de notre collection aux bactériophages peut s'expliquer par lysogénie. Cependant, pour trois autres souches très résistantes, la lysogénie ne peut pas être l'explication recherchée aux phénomènes, car nos essais n'ont pu induire la libération de particules virales.

3. L'hypothèse de l'existence de phénomènes de restriction-modification (RM) postule que tous les phages posséderaient tous les sites de reconnaissance de tous les systèmes RM rencontrés dans l'ensemble des streptocoques lactiques. Si l'on considère un phage $\alpha$ et une souche $X$, le phage $\alpha$ propagé sur sa souche $X$ aura tous les sites de reconnaissance des systèmes RM de $\mathrm{X}$ méthylés. Ces sites ainsi modifiés protégeront le phage de l'action de l'endonucléase des systèmes RM de X présents dans d'autres souches. Si ce phage est mis en contact avec une souche $\mathrm{Y}$ qui possède au plus deux systèmes $\mathrm{RM}$ que ne possède pas $\mathrm{X}$, il aura une action restreinte sur Y. Ainsi, le spectre d'action d'un phage est entièrement dépendant de la nature des systèmes RM que possède la souche de propagation. Par voie de conséquence, plus la souche de propagation a de sysmes RM, plus le phage a un large spectre d'action. Nous avons tenté 
de lever le phénomène de restriction avec la technique adoptée par Winkler et al. [8] sur les staphylocoques en chauffant les souches à $50^{\circ} \mathrm{C}-53^{\circ} \mathrm{C}$, ce qui permet de détruire l'endonucléase, tout en maintenant l'activité méthylase.

Pour S. cremoris, le temps optimal de chauffage est de $4 \mathrm{~min}$ à $50^{\circ} \mathrm{C}$; il est de $4 \mathrm{~min}$ à $53^{\circ} \mathrm{C}$ pour le $S$. lactis et le $S$. diacetylactis. Ces paramètres ont été définis pour que le pourcentage de mortalité varie entre 30 et 50 p. 100 .

Le chauffage des souches modifie peu les réactions de restriction, contrairement à ce qui a été observé sur les staphylocoques. Les groupes de lysotypie demeurent relativement stables. Le chauffage donne le moyen d'obtenir une attaque de $S$. cremoris par des phages de $S$. diacetylactis, ce qui est tout à fait nouveau par rapport aux résultats obtenus par Leclerc [4]. De même, la technique de chauffage a permis de sensibiliser des souches de $S$. diacetylactis à des phages de $S$. cremoris.

Pour des cultures très résistantes aux bactériophages, les virus obtenus de ces souches très restrictives sont virulents sur de nombreuses autres souches. Inversement, celles qui sont très sensibles aux phages sont peu restrictives et les phages obtenus sur ces cultures sont peu virulents. Seules, trois souches résistantes aux phages ne répondent pas à cette loi. On peut alors imaginer que ces cultures sont lysogènes pour un certain nombre de virus.

Les titrations de phages donnent des résultats qui passent de $10^{2}$ à $10^{4}, 10^{6}$ à $10^{8}$. Il est possible d'admettre qu'un facteur de RM fait baisser le titre de $10^{2}$. Si on admet, pour la rotation des mélanges bactériens dans l'industrie laitière, le principe suivant : "les souches de chaque mélange et d'un mélange à l'autre auront entre elles trois systèmes RM différents ", la pollution phagique se traduira par une croissance égale à $10^{2}$ virions, ce qui ne peut créer qu'un risque négligeable d'accident au cycle suivant.

\section{CONCLUSIONS}

En l'état actuel de nos connaissances, tous les streptocoques lactiques que nous avons étudiés sont capables d'adsorber les bactériophages. Il n'existe aucun groupe d'adsorption permettant de rendre compte des groupes de lysotypie.

Il est difficile d'élargir par le chauffage le spectre d'action des streptocoques lactiques. La présence de souches lysogènes permet d'expliquer la résistance de certains $S$. cremoris et $S$. lactis.

L'analyse des systèmes de restriction-modification permet d'envisager la mise en place d'une rotation efficace des souches utilisées dans l'industrie laitière. 


\section{Remerciements}

Nous tenons à remercier la Société Air Liquide pour son aide intellectuelle, matérielle et technique.

\section{S u m m a ry}

\section{STUDY OF WHEYS'S BACTERIOPHAGES}

Phage resistance of lactic mesophile streptococci is not in relation with adsorption. The adsorption do not differ from one phage to another. Lysogeny and restriction modification system are the two systems related to phage resistance. Their analysis allows strains rotation in dairy industries.

Reçu pour publication en octobre 1980.

\section{Bibliographie}

[1] Boissonnet (B.) (1977) - Etude des bactériophages des streptocoques lactiques. Thèse de Doctorat de $3^{e}$ cycle, Université de Clermont II.

[2] Bozio (J. Y.) (1976). - Etude du lysotypage et des phénomènes de restriction et de modification des streptocoques lactiques. Thèse de Doctorat $3^{\text {e }}$ cycle, Université de Clermont II.

[3] Haines (M.) and Ferreria (H. P.) (1949). - Lysogenic strains of lactic streptococci. Nature, 164, 667-668.

[4] Leclerc (M. C.) (1976). - Contrôle des levains lactiques pour l'industrie laitière. Thèse de Doctorat $3^{\mathrm{e}}$ cycle, Université de Clermont II.

[5] LowERIE (R. J.) (1974). - Lysogenic strains of group N lactic streptococci. Appl. Microb., 27, 210-217.

[6] Mac Kay (L. L.), CoRds (B. R.) and Balivius (K. A.) (1973). - Transduction of lactose metabolism in Streptococcus lactis $\mathrm{D}_{2}$. J. Bact., 116, 810-815.

[7] Rountree (P. M.) (1959). - Changes in the phage typing patterns of Staphylococci following Lysogenization. J. Gen. Microbiol., 20, 620-633.

[8] Winkler (K. C.), Stobberingh (E. E.) and Verhoef (J.) (1975). - Restriction and modification of phages in Staphylococcal phage typing Part V. Epidemiology, 376-386. 